\title{
Curvature particle pinch in tokamak and stellarator geometry
}

\author{
Alexey Mishchenko, Per Helander, Yuriy Turkin
}

\begin{abstract}
A study of the curvature pinch effect in various fusion devices (both tokamaks and stellarators) is presented. Canonical density profiles are calculated employing the theory developed in [M. B. Isichenko, A. V. Gruzinov, P. H. Diamond, and P. N. Yushmanov, Phys. Plasmas 3, 1916 (1995)]. In tokamaks, it is found that the curvature pinch is relatively strong (especially in a spherical tokamak) and usually but not always leads to a peaked density profile. In stellarators, the curvature pinch is weaker and can have either sign.
\end{abstract}

\section{Introduction}

The density profile is usually peaked in tokamaks. To explain this peaking in absence of particle sources in the plasma core the existence of an inward particle pinch (convective flux not directly associated with a density gradient) is often invoked. In neoclassical theory, such an inward pinch effect appears as an off-diagonal flux proportional to the toroidal loop voltage (the so-called Ware pinch, see Ref. [1]). This neoclassical pinch effect is typically too small to explain the experimental observations although it can be made stronger by artificially enhancing the electron-electron collision frequency [2]. Also, turbulent mechanisms can drive an anomalous pinches. Thus, so-called thermodiffusion (see Refs. $[3,4,5]$ ) driven by microinstabilities in a fusion plasma can lead to an inward particle flux proportional to the temperature gradient. However, this mechanism may not be strong enough to explain the experimentally observed density peaking (see Ref. [6]).

Another anomalous mechanism, labeled "turbulent equipartition", has been suggested (see Ref. [7]). This mechanism is associated with the adiabatic invariance of trapped electron motion. The basic physical argument is that collisionless trapped electrons experience anomalous transport through interaction with electrostatic turbulence while conserving the second adiabatic invariant constant (assuming that the characteristic frequencies of the turbulence are much smaller than the electron bounce frequency). Under such conditions, the trapped electrons which gain energy from the wave must move inwards (in a tokamak) and are adiabatically compressed to give peaked profiles. This mechanism has been 
called the curvature pinch effect $[8,9]$ (another name is turbulent equipartition pinch).

The curvature pinch has been addressed in a tokamak geometry using the large-aspect-ratio approximation [8] within a phenomenological description of the turbulent diffusion. Results valid in general tokamak geometry have also been obtained [9]. The curvature pinch has been calculated more quantitatively in a tokamak using quasilinear theory $[3,4,10]$. In stellarators, there is experimental evidence of anomalous pinches, too [11]. In Ref. [12], the possibility of a negative anomalous particle flux in a stellarator has been found numerically. There is clearly a need to gain more physical insight into the basic mechanisms which can drive stellarator pinches.

In this paper, we point out that the theory of Ref. [8] applies to stellarators as well as tokamaks and study the curvature pinch in various fusion devices, both existing and planned ones. We compute the so-called "canonical density" profile driven by the curvature pinch (see Sec. 2 for details). The results of the computations employing true geometry are compared with the corresponding large-aspect-ratio approximation.

The paper is organized as follows. In Sec. 2, we describe briefly the theory underlying our calculations and present the numerical tool that is used to calculate the canonical density in various devices. In Sec. 3, we discuss results of our calculations in a tokamak geometry, while calculations for stellarators are presented in Sec. 4. The final discussion and conclusions are given in Sec. 5.

\section{Basic theory of the curvature pinch}

In this section, our presentation and notation closely follow Ref. [8]. If the electron bounce frequency is larger than the turbulence correlation frequency, $\omega_{b} \gg \omega$, the single-particle motion can be averaged before introducing the kinetic description. Using the Clebsch coordinates $\psi$ (poloidal magnetic flux) and $\alpha$ (corresponding angle) defined locally for a general magnetic field $\mathbf{B}(\mathbf{x})=$ $\nabla \alpha \times \nabla \psi /(2 \pi)$, one can write the equations of bounce-averaged guiding center motion in the canonical Hamiltonian form:

$$
\dot{\psi}=\frac{2 \pi c}{e}\left(\frac{\partial H_{0}}{\partial \alpha}+e \frac{\partial \tilde{\phi}}{\partial \alpha}\right), \quad \dot{\alpha}=-\frac{2 \pi c}{e}\left(\frac{\partial H_{0}}{\partial \psi}+e \frac{\partial \tilde{\phi}}{\partial \psi}\right)
$$

Here $\tilde{\phi}$ is the fluctuating part of the bounce-averaged electrostatic potential (turbulence) and $H_{0}=\left\langle m v_{\|}^{2} / 2+\mu B+e \phi_{0}\right\rangle_{b}$ is the bounced-averaged unperturbed particle Hamiltonian function with $\phi_{0}$ being the background electric field.

These equations can be interpreted as Langevin equations with the stochastic part coming from the turbulence. According to the theory of stochastic processes, these Langevin equations for the particle motion can equivalently be rewritten as the Fokker-Planck equation for the particle distribution function:

$$
\frac{\partial}{\partial t} f(\psi, \alpha)=-\partial_{i}\left[A^{i} f(\psi, \alpha)\right]+\partial_{i}\left[D^{i j} \partial_{j} f(\psi, \alpha)\right]+C[f(\psi, \alpha)], \quad i=(\psi, \alpha),
$$


where the derivatives are taken at constant values of the first and second adiabatic invariants, $\mu=v_{\perp}^{2} /(2 B)$ and $J=\oint v_{\|} \mathrm{d} l$. Note that the collision integral $C(f)$ has been included in Eq. (2). One can further reduce the description of the system by averaging over $\alpha$ [which annihilates all terms of the type $\partial_{\alpha}(\ldots)$ ]. Thus, the reduced Fokker-Planck equation takes the form:

$$
\frac{\partial}{\partial t}\langle f\rangle_{\alpha}=-\partial_{\psi}\left\langle A^{\psi} f\right\rangle_{\alpha}+\partial_{\psi}\left\langle D^{\psi \psi} \partial_{\psi} f\right\rangle_{\alpha}+\langle C(f)\rangle_{\alpha},
$$

Note that in addition to a pure diffusion in the $\psi$-space (as it is the case in the theory developed in Ref. [8]), a drift term appears in Eq. (3). This term matters only in non-axisymmetric configurations [otherwise the drift coefficient $A^{\psi}=(2 \pi c / e) \partial_{\alpha} H_{0}$ vanishes $]$.

Formally, one seeks the solution of Eq. (3) by successive approximations in the smallness of $D^{\psi \psi}$ (turbulence energy is smaller than thermal energy) and $A^{\psi}$ (neoclassical ordering). The zeroth-order solution follows from the condition $C\left(f_{0}\right)=0$ and is given by the expression:

$$
f_{0}(\psi, \mu, \mathcal{B})=\frac{n(\psi)}{\sqrt{2 \pi m} T^{3 / 2}(\psi)} \exp \left[-\frac{m \mu \mathcal{B}}{T(\psi)}\right],
$$

where $\mathcal{B}=\left(\epsilon-e \phi_{0}\right) /(m \mu)$ is the maximal magnetic field on a flux surface which is available for an electron with energy $\epsilon=m v^{2} / 2+e \phi_{0}$ and magnetic moment $\mu=v_{\perp}^{2} /(2 B)$.

In first order, Eq. (3) can be written as follows:

$$
\partial_{t}\left\langle f_{0}\right\rangle_{\alpha}=\underbrace{-\partial_{\psi}\left\langle A^{\psi} f_{0}\right\rangle_{\alpha}+\left\langle C\left(f_{1}\right)\right\rangle_{\alpha}}_{\text {neoclassical }}+\underbrace{\partial_{\psi}\left\langle D^{\psi \psi} \partial_{\psi} f_{0}\right\rangle_{\alpha}}_{\text {anomalous }},
$$

The first two terms in Eq. (5) lead to neoclassical fluxes (their effect should be relatively small in a tokamak). The last term corresponds to anomalous diffusion and pinch.

We can construct an equation for the density moment integrated over the flux surface, using the relation:

$$
\left\langle\int \mathrm{d}^{3} v F\right\rangle=\underbrace{\frac{1}{V^{\prime}} \sum_{\sigma} \int \mathrm{d} \mu \mathrm{d} J F_{\sigma}}_{\text {passing particles }}+\underbrace{\frac{1}{V^{\prime}}\left\langle\int \mathrm{d} \mu \mathrm{d} J F\right\rangle_{\alpha}}_{\text {trapped particles }},
$$

where $\langle\ldots\rangle$ is the flux-surface average, $\langle\ldots\rangle_{\alpha}=\oint \mathrm{d} \alpha(\ldots)$ corresponds to the average over the toroidal angle, $V^{\prime}=\mathrm{d} V / \mathrm{d} \psi$ is the derivative of the magnetic volume inside the flux surface, and $\sigma= \pm 1$ corresponds to the direction of the parallel velocity of a passing particle. For passing particles, we define $J=$ $V^{\prime}\left\langle 2 \pi B\left|v_{\|}\right|\right\rangle$. For trapped particles, $J=\oint v_{\|} \mathrm{d} l$ where the integral is taken along the magnetic field line between the bounce points (forth and back). The magnetic moment is $\mu=v_{\perp}^{2} /(2 B)$. 
The resulting continuity equation takes the form:

$$
\partial_{t} N_{e}=-\partial_{\psi}\left(\Gamma_{\text {neo }}+\Gamma_{\text {anom }}\right),
$$

where $N_{e}=V^{\prime} \int f_{0} \mathrm{~d}^{3} v$ is the per-unit-flux density, $\Gamma_{\text {neo }}$ is the neoclassical particle flux which results from the first two terms in Eq. (5), and the anomalous flux is given by the expression:

$$
\Gamma_{\text {anom }}=-\left\langle\int \mathrm{d} \mu \mathrm{d} J D^{\psi \psi}\left(\partial_{\psi} f_{0}\right)\right\rangle_{\alpha}
$$

In Eq. (8), only the trapped-electron anomalous flux is included (see the comment on passing electrons below). Following Ref. [8], we introduce the bounceinvariant pitch-angle variable $j=J / \sqrt{\mu}=\int \mathrm{d} l \sqrt{(\mathcal{B}-B) / 2}$ and expand the $\mu$-dependence of the turbulent-diffusion coefficient in a Taylor series:

$$
D^{\psi \psi}(\mu, j, \psi, \alpha)=\sum_{l=0}^{\infty} D_{l}(j, \psi, \alpha) \mu^{l} / l !
$$

Taking the derivative of the zero-oder distribution function Eq. (4) with respect to $\psi$ at constant $j$ and $\mu$, one can rewrite the anomalous flux as follows:

$$
\begin{aligned}
\Gamma_{\text {anom }}= & -\left\langle\sum_{l=0}^{\infty} \frac{(2 l+1) ! !}{\sqrt{8}(2 l) ! !} \int_{0}^{\infty} \frac{D_{l} \mathrm{~d} j}{\mathcal{B}^{3 / 2}}\left(\frac{T}{\mathcal{B}}\right)^{l} 2 n \times\right. \\
& {\left.[\underbrace{\frac{\partial \ln n}{\partial \psi}}_{\text {diffusion }}+\underbrace{l \frac{\partial \ln T}{\partial \psi}}_{\text {thermodiffusion }}-\underbrace{(l+3 / 2) \frac{\partial \ln \mathcal{B}}{\partial \psi}}_{\text {curvature pinch }}]\right\rangle_{\alpha} }
\end{aligned}
$$

This equation is valid in stellarators as well as in tokamaks. One sees that different contributions to the anomalous flux include diffusion driven by the density gradient, thermodiffusion driven by the temperature gradient and the curvature pinch, which is an effect of magnetic geometry. In contrast with $\nabla n$ and $\nabla T$, the magnetic geometry is not a thermodynamic force and the corresponding flux is not constrained by Onsager symmetry. Note that as a result of the curvature pinch, the plasma relaxes toward some profiles $n_{\text {can }}(\psi)$ and $T_{\text {can }}(\psi)$ (called "canonical profiles" in this context) instead of flat profiles, which would result from fluxes proportional to the temperature and density gradients only. In Ref. [10], it is shown that the true thermodynamic forces which have to be used in the transport calculation are the gradients of the density and temperature normalized to their canonical values. It has been shown that for such a formulation Onsager symmetry holds (at least in the quasilinear approximation) and the corresponding transport matrix is consistent with the second principle of thermodynamics (positivity of the entropy production rate).

Here, we study the curvature pinch using the canonical density profile as its main characteristics. This is determined by the magnetic configuration only, so 
that we can compare curvature pinches in various fusion devices (tokamaks as well as stellarators). Following Ref. [8], we truncate the series Eq. (10) at zero order $l=0$. Then, the anomalous particle flux takes the form:

$$
\Gamma_{\text {anom }}=-\hat{D} \frac{\mathrm{d} n}{\mathrm{~d} \psi}+\hat{V} n
$$

where the anomalous diffusion coefficient and the pinch velocity are given by the expressions:

$$
\hat{D}(\psi)=\frac{1}{\sqrt{8}}\left\langle\int \mathrm{d} j D_{0} \mathcal{B}^{-3 / 2}\right\rangle_{\alpha}, \hat{V}(\psi)=\frac{3}{2 \sqrt{8}}\left\langle\int \mathrm{d} j D_{0} \mathcal{B}^{-3 / 2} \partial_{\psi} \ln \mathcal{B}\right\rangle_{\alpha}
$$

The canonical density results from the condition $\Gamma_{\text {anom }}=0$ and can be written as follows:

$$
n_{\text {can }}(\psi)=n(0) \exp \left\{\int_{0}^{\psi} \frac{\hat{V}(\psi)}{\hat{D}(\psi)} \mathrm{d} \psi\right\}
$$

The form of the canonical profile defines if the direction of the pinch is inward (peaked canonical profile $\mathrm{d} n_{\text {can }} / \mathrm{d} \psi>0$ ) or outward (hollow canonical profile $\left.\mathrm{d} n_{\text {can }} / \mathrm{d} \psi<0\right)$.

In Ref. [8], a simplified assumption for the turbulent diffusion coefficient was made:

$$
D_{0}(j, \psi)=\Theta\left[j_{c}(\psi)-j\right] D_{0}(\psi)
$$

where $\Theta(x)$ is the Heaviside function and the quantity $j_{c}(\psi)=j\left(\psi, B_{\max }\right)=$ $\int \mathrm{d} l \sqrt{\left(B_{\max }-B\right) / 2}$ defines the boundary between the trapped and passing electrons on a given flux surface $\left[B_{\max }(\psi)\right.$ is the maximal magnetic field on the surface $\psi$ ]. The assumption Eq. (14) implies that all trapped electrons react on the turbulence in the same way, whereas passing electrons are not included into the consideration. The basic physical argument is as follows. The passing electrons are assumed to have, on average, a weaker interaction with the electrostatic turbulence since they have no turning points and instead average over all phases of the waves as they complete multiple circuits around the torus. The intention of this paper is to study the canonical density profile driven by the curvature pinch (recall that this profile corresponds to the case where the diffusion is exactly balanced by the pinch). However, this mechanism acts neither on the ions nor on the impurities (whose second adiabatic invariant is not conserved by the turbulence). Thus, anomalous transport of the trapped electrons is the dominant factor which determines the canonical density profile whereas passing electrons, ions and impurities adjust their transport to follow and ensure ambipolarity. Of course, one may raise various objections against these arguments. However, it is not the intention of the present paper to delve into this issue further, but simply to accept this approximation as reasonable enough (following Refs. [7, 8, 9]) and instead explore how its consequences depend on the magnetic geometry.

Employing Eq. (14) for the particle diffusion coefficient $D_{0}(j, \psi)$ one can explicitly calculate the integrals appearing in Eq. (12). The canonical density 
resulting from this calculation can be written as follows:

$n_{\text {can }}(\rho)=n_{0} \exp \left\{\int_{0}^{\rho} \mathrm{d} \rho\left[-\frac{\iota}{V_{s}^{\prime} I_{1 / 2}} \frac{\mathrm{d}}{\mathrm{d} \rho}\left(\frac{V_{s}^{\prime} I_{3 / 2}}{\iota}\right)+\frac{3}{2} \frac{1}{B_{\max }} \frac{\mathrm{d} B_{\max }}{\mathrm{d} \rho}\left(1-\frac{I_{3 / 2}}{I_{1 / 2}}\right)\right]\right\}$

Here, we use the quantity $\rho=\sqrt{s}$ as the flux surface label with $s=\chi / \chi_{a}$ being the normalized toroidal flux through given flux surface ( $\chi$ is the toroidal flux on a given flux surface, $\chi_{a}$ is the toroidal flux on the edge). Also, we have introduced the following notations:

$$
I_{1 / 2}(\rho)=\left\langle\sqrt{1-B / B_{\max }}\right\rangle, \quad I_{3 / 2}(\rho)=\left\langle\left(1-B / B_{\max }\right)^{3 / 2}\right\rangle, \quad V_{s}^{\prime}(\rho)=\frac{\mathrm{d} V}{\mathrm{~d} s}
$$

with \langle\rangle being the flux-surface average. Other quantities are the rotational transform $\iota(\rho)=\mathrm{d} \psi / \mathrm{d} \chi(\psi$ is the poloidal flux $)$, maximal magnetic field on a given flux surface $B_{\max }(\rho)$ and the volume inside the flux surface $V(\rho)$. Note that Eq. (15) includes only flux functions and can be used in tokamaks as well as in stellarators.

The derivative $V^{\prime \prime}(\rho)$ appears in the first term of Eq. (15). We shall see that this quantity is important for the form of the canonical density profile. Thus, configurations with a magnetic well $\left(V^{\prime \prime}>0\right)$ tend to have peaked canonical density profiles whereas configuration with a magnetic hill $\left(V^{\prime \prime}<0\right)$ may have hollow profiles. Note also that diamagnetic effects due to finite beta may influence the density profile [affecting both $V^{\prime \prime}$ and the maximal magnetic field on a flux surface $\left.B_{\max }(\rho)\right]$. Flux-surface shaping and effects of 3D geometry enter into the canonical density profile through the quantities $I_{1 / 2}$ and $I_{3 / 2}$.

For a large-aspect-ratio tokamak with unshifted circular flux surfaces one can simplify Eq. (15). In this case, the magnetic field strength can be written as follows:

$$
B(\rho)=B_{0}\left[1-\frac{r_{a}}{R_{0}} \rho \cos \theta+\mathcal{O}\left(\frac{r_{a}^{2}}{R_{0}^{2}}\right)\right], \rho \in[0 ; 1]
$$

with $R_{0}$ the major radius, $r_{a}$ the minor radius and $\rho=r / r_{a}$. Substituting this expression into Eq. (15), one can derive the large-aspect-ratio limit for the canonical density:

$$
n_{\text {can }}(\rho) \approx n_{0}(0)\left[1-\frac{4 r_{a}}{3 R_{0}} \int_{0}^{\rho} \mathrm{d} \rho\left(\frac{\mathrm{d} \ln q}{\mathrm{~d} \ln \rho}+\frac{3}{8}\right)+\mathcal{O}\left(\frac{r_{a}^{2}}{R_{0}^{2}}\right)\right]
$$

This result is sometimes used in fluid turbulence simulations of particle transport (see Refs. [10,3,13]), although it tends to overestimate the curvature pinch, as we shall see. In fact, Eq. (18) can lead to negative densities, if the magnetic shear is large. Thus, it is more reasonable to define the large-aspect-ratio limit 
for the canonical density as follows:

$$
n_{\text {can }}(\rho) \approx n_{0}(0) \exp \left[-\frac{4 r_{a}}{3 R_{0}} \int_{0}^{\rho} \mathrm{d} \rho\left(\frac{\mathrm{d} \ln q}{\mathrm{~d} \ln \rho}+\frac{3}{8}\right)\right]+\mathcal{O}\left(\frac{r_{a}^{2}}{R_{0}^{2}}\right)
$$

To compute the flux-surface averages appearing in Eq. (15) we use the equilibrium code package MConf (standing for Magnetic CONFiguration). The main purpose of this package is to provide fast and convenient tool for coordinate transformations between Boozer magnetic coordinates and real space coordinates. Furthermore, MConf calculates various information about the magnetic configuration of a stellarator or a tokamak, such as the magnetic field, the Jacobian, rotational transform $(\iota)$, trapped particle fraction, minimum and maximum magnetic field on a flux surface, the volume inside each flux surface, etc.

\section{Tokamak results}

ITER (International Thermonuclear Experimental Reactor [14]). The canonical density profile for ITER has been calculated in Ref. [9], which provides a useful benchmark on our numerical tool. Note that the strength of the curvature pinch is much weaker than one would expect from the large-aspect-ratio approximation (see Fig. 1).

MAST (Mega-Ampere Spherical Tokamak [15], operated at Culham, UKAEA). This configuration is of particular interest in this context because the canonical density driven by the diffusion of trapped electrons [see Eq. (15)] is an effect of order $\epsilon=r_{a} / R_{0}$, which should be of order unity in a spherical tokamak (in MAST $\epsilon \approx 0.68$ ). In Fig. 2 one sees that the density profile is indeed much more peaked than in ITER (which implies a stronger curvature pinch). The large-aspect-ratio approximation Eq. (19) cannot be used as it overestimates the strength of the pinch. The canonical density even becomes negative if Eq. (18) is used (MAST has very high magnetic shear at the plasma edge).

NSTX (National Spherical Torus Experiment [16], Princeton). In Fig. 3, one can see that, in contrast with other tokamaks, the canonical density profile corresponding to this configuration is non-monotonic and shows off-axis peaking. The reason is that the case considered here is characterized by high pressure and large pressure gradient which lead to appearance of a magnetic well off-axis (at $\rho \approx 0.5)$ and a magnetic hill in the center $(\rho=0)$. We have here an example of a finite-beta effect on the curvature pinch in a tokamak. In the following, we will see that there are finite-beta effects on the curvature pinch in stellarators, too. It is not surprising, that the large-aspect-ratio approximation Eq. (19) leads to a qualitatively wrong form of the canonical density profile. 


\section{Stellarator results}

NCSX (National Compact Stellarator Experiment [17], under construction in Princeton). This quasi-axisymmetric stellarator exhibits a hollow canonical density profile (see Fig. 4), corresponding to an outward curvature pinch, which is in contrast to the inward curvature pinch observed in tokamak configurations. One can see that the curvature pinch is relatively weak, but still stronger than that in other stellarator devices, see below. This is caused by the relatively small aspect ratio of NCSX. Note that the "equivalent tokamak" (a circular tokamak with the same aspect ratio and rotational transform as the stellarator) would have a nearly flat (slightly peaked) canonical density profile (see Fig. 4). Thus, the appearance of a hollow profile cannot be explained by the stellarator shear reversal only but incorporates effects of flux-surface shaping and 3D geometry, too.

LHD (Large Helical Device operated at NIFS, Japan, see Ref. [18]). One of the attractive characteristics of LHD is that different configurations can be realized by changing coil currents. These configurations can be characterized by the position of the vacuum magnetic axis. In typical LHD plasmas (standard inward shifted configuration with $R_{\mathrm{ax}}=3.75 \mathrm{~m}$ ), the experimentally observed density has a hollow profile (see Ref. [11]) whereas the density profiles tend to be flat [11] in a configuration with $R_{\mathrm{ax}}=3.53 \mathrm{~m}$, which is the neoclassically optimized configuration (see Ref. [19]). This effect has been addressed in Ref. [12], where the existence of both positive and negative anomalous particle fluxes was obtained depending on the choice of parameters.

In Fig. 5, we consider the curvature pinch effect in the standard inward shifted configuration $\left(R_{\mathrm{ax}}=3.75 \mathrm{~m}\right)$. This configuration shows nearly flat density profile. The hollow profiles observed in this configuration experimentally can be attributed to neoclassical fluxes as stated in Refs. [11, 12]. The neoclassically optimized configuration $\left(R_{\mathrm{ax}}=3.53 \mathrm{~m}\right)$ has a canonical density profile which is peaked in the center and hollow at the edge (see Fig. 6). Thus, an inward curvature pinch becomes effective only for particles with $\rho \leq 0.7$.

Note that this feature may have a positive influence on impurity transport. In Ref. [13], it was concluded on the basis of a fluid model that impurity ions respond to the curvature pinch in much the same way as do the bulk ions. Thus, impurities accumulate in the center of the device if the curvature pinch is inward for all radial positions (as it is usually the case in a tokamak). In the case shown in Fig. 6, however, impurities have to penetrate well inside the device due to some additional mechanism before they are pushed further inwards by the curvature pinch. Thus, the curvature pinch could perhaps prevent impurity accumulation in the center while still allowing density peaking in stellarators. This is in contrast with tokamak case where the curvature pinch is always a driving factor for the impurity accumulation in the plasma core (see Ref. [13]).

W7-AS. Next, we consider the stellarator Wendelstein 7-AS (see Ref. [20]) which was operated in Garching, Germany. This stellarator has been partially 
optimized (strong reduction of Pfirsch-Schlüter current). In Fig. 7, we plot the canonical density corresponding to the so-called standard configuration (low $\beta$, $\iota=1 / 3$ ). One sees that the density is slightly peaked but the curvature pinch is very weak (which can be explained by the very low shear and large aspect ratio of the device). Note that, as usual, the density profile corresponding to the "equivalent tokamak" [i.e. computed using Eq. (19)] deviates considerably from the actual canonical density profile [computed using Eq. (15)], which again demonstrates the role of the flux-surface shaping and 3D geometry in the formation of the canonical density profile in stellarators.

In Fig. 8, the canonical density corresponding to the W7-AS configuration with $\beta=0.0165$ is plotted. One can see that a magnetic well appears as a finite-beta effect. The density profile becomes hollow (5\% density increase on the edge), which indicates an outward curvature pinch. Note that the pinch is now stronger than in the previous (low-beta) case. Thus, finite-beta effects may influence the strength of the curvature pinch and even change its direction.

W7-X. The last device to be considered is the fully optimized stellarator Wendelstein 7-X (under construction in Greifswald, see Ref. [21]). In Fig. 9, the so-called standard (low-beta) configuration is studied. One sees that the density is nearly flat (slightly hollow). The curvature pinch becomes much stronger in the case of the high-beta configuration (see Fig. 10 where $\beta=0.0448$ ). The resulting canonical density profile is hollow (as in the high-beta W7-AS) which may inhibit the impurity accumulation in the core plasma.

\section{Conclusions}

In this paper, we have shown that the curvature pinch affects both tokamaks and stellarators. This pinch effect can be characterized by the corresponding canonical density profile, which has been computed for various fusion devices.

The curvature pinch is usually inward in tokamak geometry and its strength is proportional to the inverse aspect ratio. The effect is therefore much larger in a spherical tokamak such as MAST than in ITER. An especially interesting result is obtained from NSTX where the canonical density profile is non-monotonic and exhibits off-axis peaking (caused by the off-axis magnetic well which appears as a finite-beta effect). Hence, turbulent equipartition does not always lead to a central density peaking in tokamak geometry which contrasts with widespread believe.

In stellarators, the curvature pinch is rather weak. The main reason for this is the large aspect ratio and negative magnetic shear characterizing most stellarators. Nevertheless, the curvature pinch may play a role in stellarators, too. A qualitative difference to tokamaks is that the curvature pinch in most cases leads to hollow density profiles. This may inhibit impurity accumulation in the core. Clearly, one has to take into account also the neoclassical particle transport of electrons which is non-negligible in stellarators.

Note that the computation of the canonical density profile (as the "quantity 
of reference" which has to be used in the transport equations, see Ref. [10]) is important in its own right. We have limited ourselves to this effect only, leaving aside the possible role of thermodiffusion. In tokamak geometry, the relative importance of the thermodiffusion is a question of debate (see Refs. [3, 10, 5, 13]). In stellarators, the role of thermodiffusion merits further study. It is of course possible that it is more important than the curvature pinch, which, as we have seen, is rather weak in most stellarators.

We have summarized our results in Figs. 11 and 12, where the densitypeaking factor, defined as the normalized difference between the maximal and minimal values of the canonical density, is plotted as a function of the inverse aspect ratio. One sees that the peaking in tokamaks increases with the inverse aspect ratio, whereas the peaking in stellarators does not. In Fig. 12, one sees that the curvature pinch leads to different density profiles in the same stellarator device depending on the particular magnetic configuration used. An interesting feature is the beta-dependence of the curvature pinch strength and even its direction (both in tokamaks and stellarators).

The curvature pinch appears because collisionless trapped electrons experience anomalous transport through interaction with electrostatic turbulence while maintaining their second adiabatic invariant. This assumes that the characteristic frequencies of the turbulence are much smaller than the electron bounce frequency. Note that this condition can be satisfied for fast ions, too, so that one may expect a fast-ion curvature pinch in addition to any anomalous diffusion that may arise from the interaction between the fast ions and the turbulence fluctuations. This may be of importance in view of recent numerical results $[22,23]$ indicating finite levels of anomalous fast-ion transport.

\section{ACKNOWLEDGMENTS}

We appreciate useful discussions with J. Nührenberg, M. Mikhailov and N. Marushchenko. We acknowledge S. Kaye for his help on NSTX.

\section{References}

[1] A. A. Ware, Phys. Rev. Lett. 25, 15 (1970).

[2] G. V. Pereverzev and P. N. Yushmanov, Sov. J. Plasma Phys. 6, 543 (1980).

[3] F. Miskane, X. Garbet, A. Dezairi, and D. Saifaoui, Phys. Plasmas 7, 4197 (2000).

[4] D. R. Baker, Phys. Plasmas 11, 992 (2004).

[5] P. W. Terry and R. Gatto, Phys. Plasmas 13, 062309 (2006). 
[6] F. Wagner and U. Stroth, Plasma Phys. Controlled Fusion 35, 1321 (1993).

[7] J. Nycander and V. V. Yankov, Phys. Plasmas 2, 2874 (1995).

[8] M. B. Isichenko, A. V. Gruzinov, P. H. Diamond, and P. N. Yushmanov, Phys. Plasmas 3, 1916 (1995).

[9] D. R. Baker and M. N. Rosenbluth, Phys. Plasmas 5, 2936 (1998).

[10] X. Garbet, N. Dubuit, E. Asp, Y. Sarazin, C. Bourdelle, P. Chendrin, and G. H. Hoang, Phys. Plasmas 12, 082511 (2005).

[11] K. Tanaka, C. Michael, M. Yokoyama, O. Yamagishi, K. Kawahata, and T. Tokuzawa, Fusion Sci. Technol. 51, 97 (2007).

[12] O. Yamagishi, M. Yokoyama, N. Nakajima, and K. Tanaka, Phys. Plasmas 14, 012505 (2007).

[13] N. Dubuit, X. Garbet, T. Parisot, R. Guirlet, and C. Bourdelle, Phys. Plasmas 14, 042301 (2007).

[14] R. Aymar, Fusion Eng. Des. 36, 9 (1997).

[15] A. Sykes, J.-W. Ahn, R. Akers et al., Phys. Plasmas 8, 2101 (2001).

[16] S. Kaye, M. Ono, Y.-K. M. Peng, D. B. Batchelor, M. D. Carter, W. Choe, and R. Goldston, Fusion Technol. 36, 16 (1999).

[17] A. Reiman, G. Fu, S. Hirshman et al., Plasma Phys. Controlled Fusion 41, B273 (1999).

[18] O. Motojima, N. Ohyabu, A. Komori et al., Nucl. Fusion 43, 1674 (2003).

[19] S. Murakami, A. Wakasa, H. Maaberg, C. D. Beidler, H. Yamada, K. Y. Watanabe, and LHD Experimental Group, Nucl. Fusion 42, L19 (2002).

[20] G. Grieger, W. Lotz, P. Merkel et al., Phys. Fluids 4, 2081 (1992).

[21] G. Grieger, C. D. Beidler, H. Maassberg et al., in Proceedings of the 13th International Conference on Plasma Physics and Controlled Nuclear Fusion Research (International Atomic Energy Agency, Vienna, 1991), Vol. 3, p. 525 .

[22] T. Hauff and F. Jenko, Phys. Plasmas 13, 102309 (2006).

[23] C. Estrada-Mila, J. Candy, and R. E. Waltz, Phys. Plasmas 13, 112303 (2006).

[24] M. Otte, O. Lischtschenko, S. Marsen, M. Schubert, Y. Y. Podoba, F. Wagner, G. B. Warr, L. I. Krupnik, A. V. Melnikov, and A. I. Zhezhera, Stellarator News 106, 4 (2006). 


\section{Figure captions}

Fig. 1: ITER (inverse aspect ratio $\epsilon \approx 0.38$ ). Left: rotational transform $\iota$, normalized flux derivative of the magnetic volume $\left(V^{\prime}=\mathrm{d} V / \mathrm{d} \psi\right.$ where $\psi$ is the poloidal flux), normalized maximal magnetic field on a surface $B_{\max }$ as functions of $\rho=\sqrt{\chi / \chi_{a}}$ with $\chi$ being toroidal flux. Right: canonical density computed using Eq. (15) (solid line) vs. large-aspect-ratio limit given by Eq. (19) (dashed line). Color online.

Fig. 2: MAST (inverse aspect ratio $\epsilon \approx 0.68$ ). Curves as in Fig. 1. Color online.

Fig. 3: NSTX (inverse aspect ratio $\epsilon \approx 0.6$ ). Curves as in Fig. 1. Color online.

Fig. 4: NCSX (inverse aspect ratio $\epsilon \approx 0.22$ ). Curves as in Fig. 1. Color online.

Fig. 5: LHD (inward-shifted configuration with position of magnetic axis $R_{\mathrm{ax}}=$ $3.75 \mathrm{~m}$, inverse aspect ratio $\epsilon \approx 0.15$ ). Curves as in Fig. 1. Color online.

Fig. 6: LHD (neoclassically optimized configuration with position of magnetic axis $R_{\mathrm{ax}}=3.53 \mathrm{~m}$, inverse aspect ratio $\epsilon \approx 0.17$ ). Curves as in Fig. 1. Color online.

Fig. 7: W7-AS (standard configuration, inverse aspect ratio $\epsilon \approx 0.09$ ). Curves as in Fig. 1. Color online.

Fig. 8: W7-AS (finite-beta configuration, inverse aspect ratio $\epsilon \approx 0.09$ ). Curves as in Fig. 1. Color online.

Fig. 9: W7-X (standard configuration, inverse aspect ratio $\epsilon \approx 0.09$ ). Curves as in Fig. 1. Color online.

Fig. 10: W7-X (finite-beta configuration, inverse aspect ratio $\epsilon \approx 0.09$ ). Curves as in Fig. 1. Color online.

Fig. 11: Peaking factor $\left(n_{\max }-n_{\min }\right) / n_{\max }$ as a function of inverse aspect ratio, both tokamak and stellarator results. Note that a large-aspect-ratio tokamak (inverse aspect ratio $\epsilon=0.05$ ) with circular unshifted flux surfaces has been considered in addition to the cases described in the text. Color online.

Fig. 12: Peaking factor $\left(n_{\max }-n_{\min }\right) / n_{\max }$ as a function of inverse aspect ratio, stellarator results. Note that a classical $l=2$ stellarator WEGA [24], the LHD configurations with $R_{\mathrm{ax}}=4.05 \mathrm{~m}$ and $R_{\mathrm{ax}}=3.90 \mathrm{~m}$, low- and high- mirror W7$\mathrm{X}$ configurations have been considered in addition to the stellarators described in Sec. 4. Color online. 

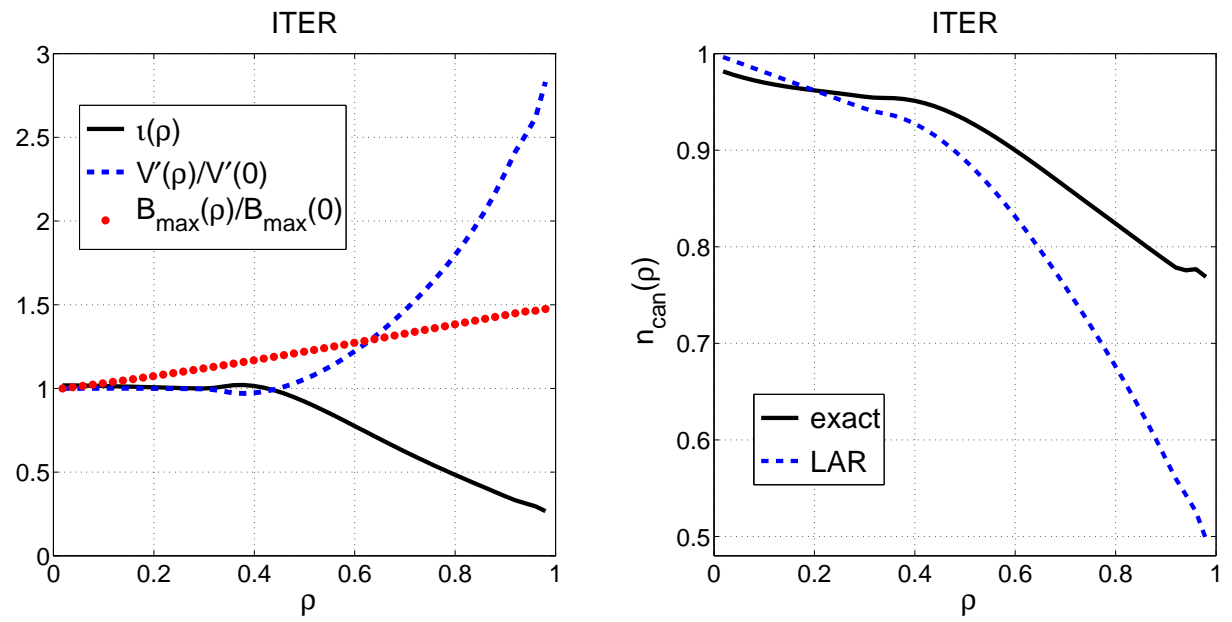

Figure 1: Mishchenko et al. 

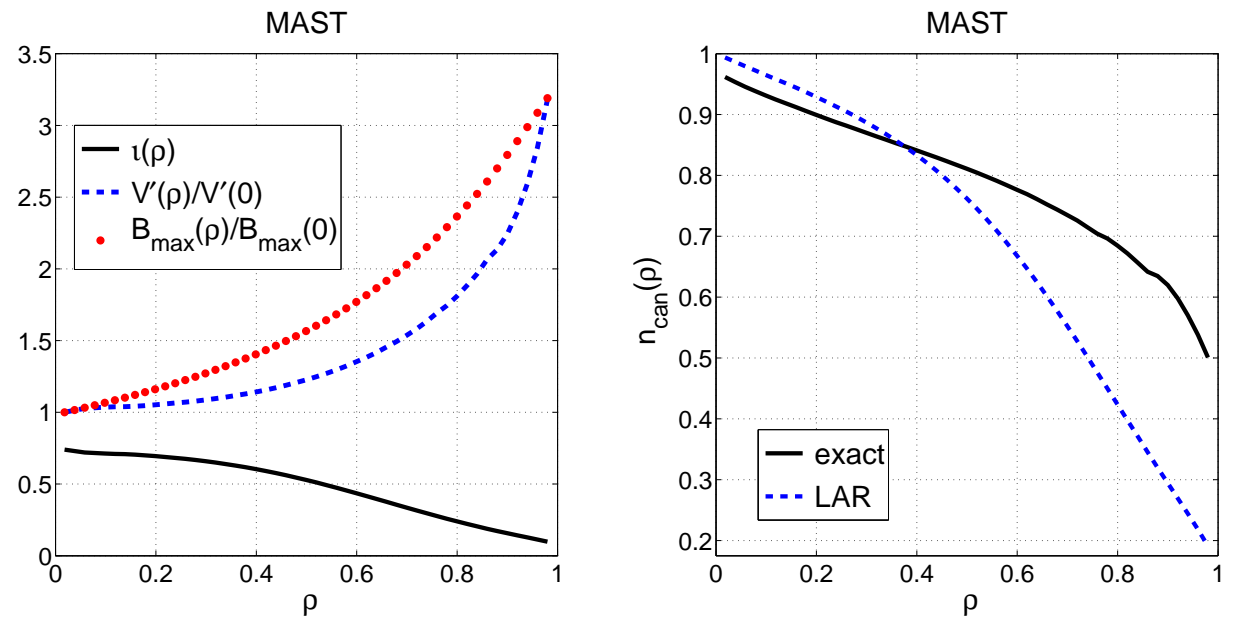

Figure 2: Mishchenko et al. 

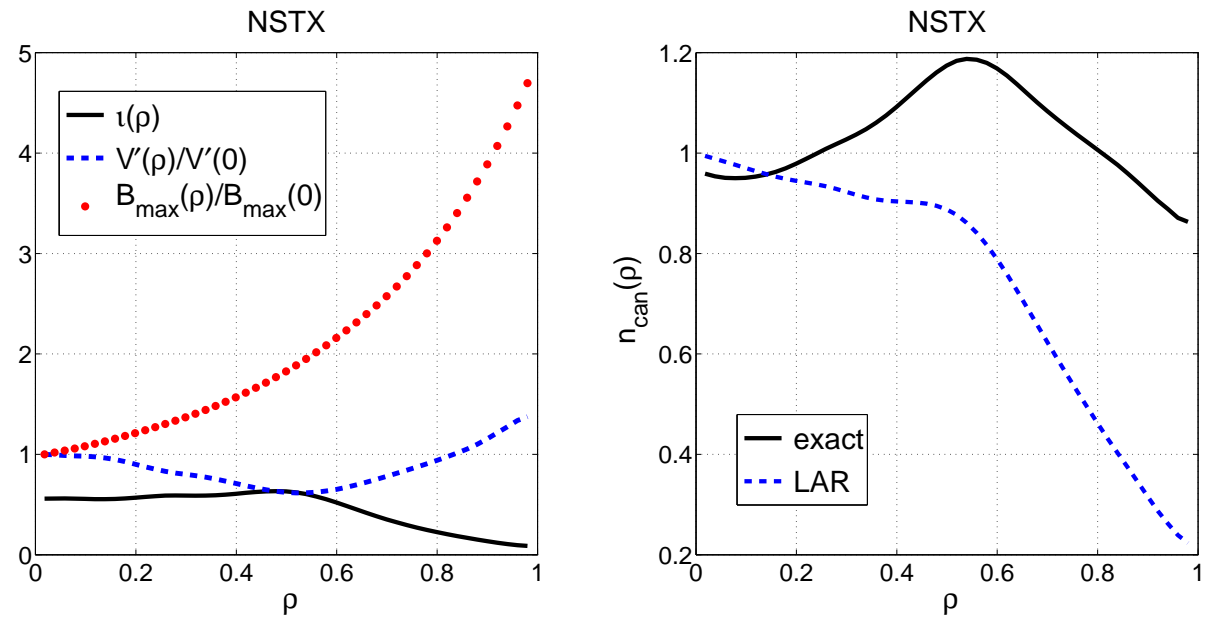

Figure 3: Mishchenko et al. 

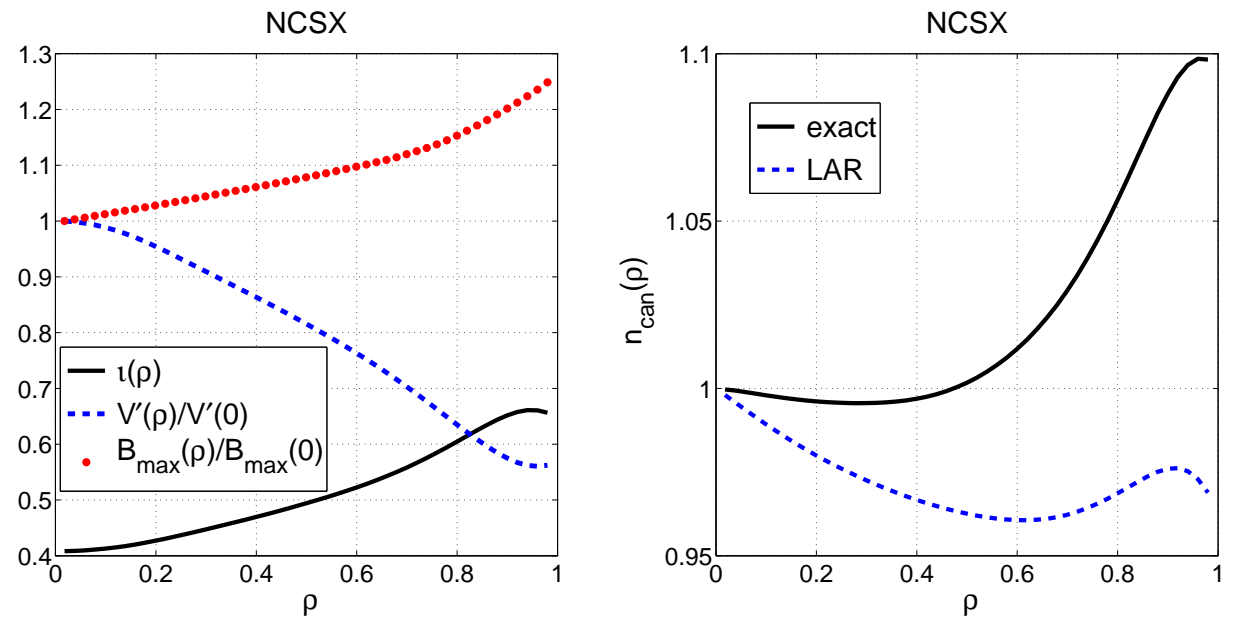

Figure 4: Mishchenko et al. 

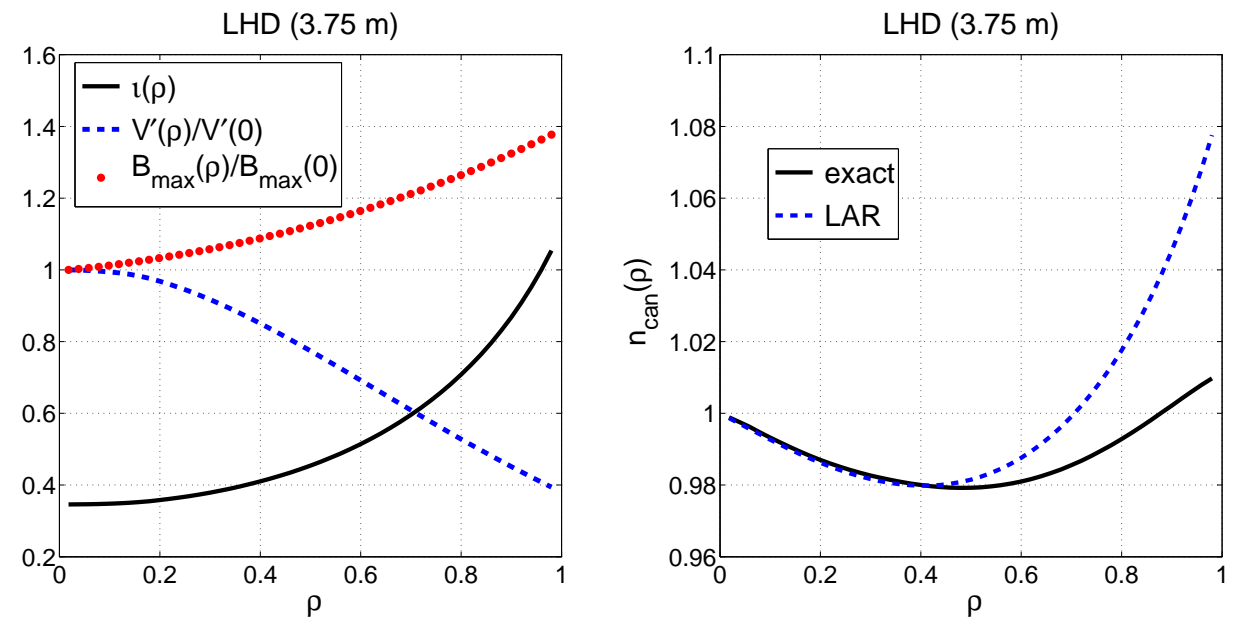

Figure 5: Mishchenko et al. 

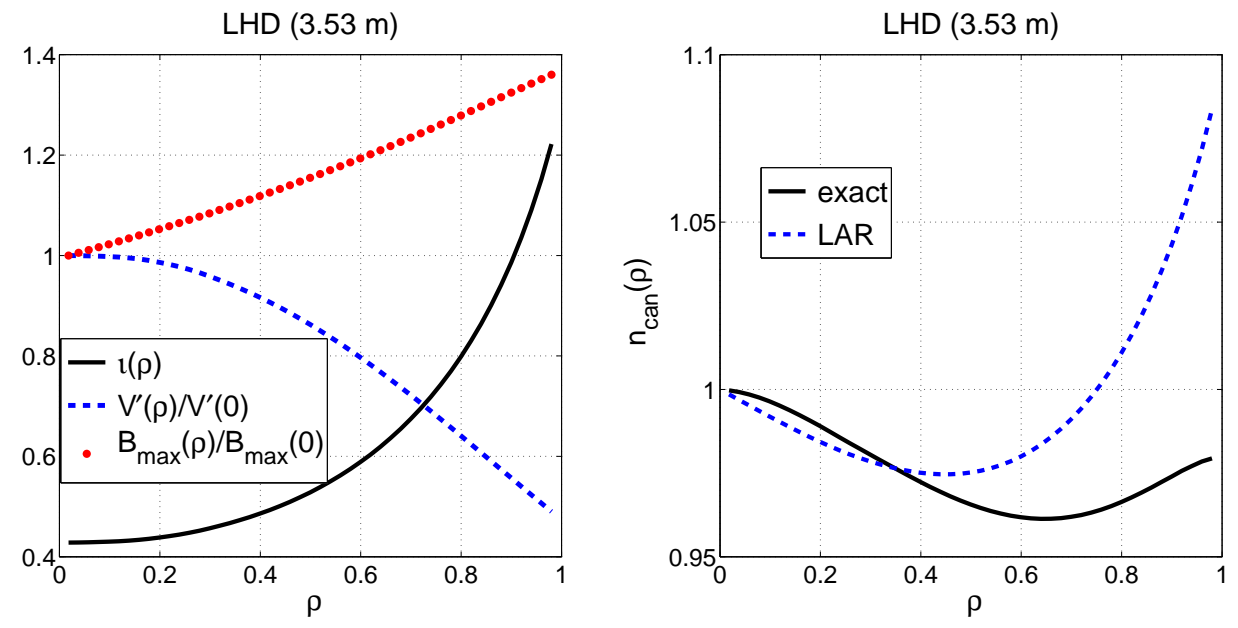

Figure 6: Mishchenko et al. 

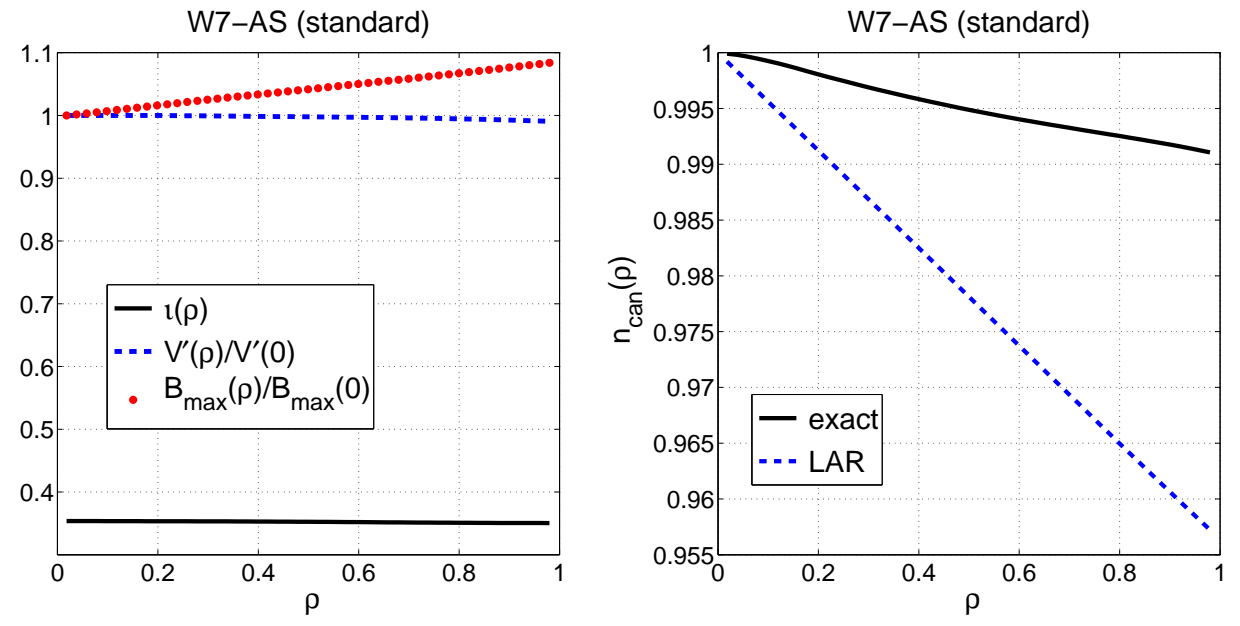

Figure 7: Mishchenko et al. 

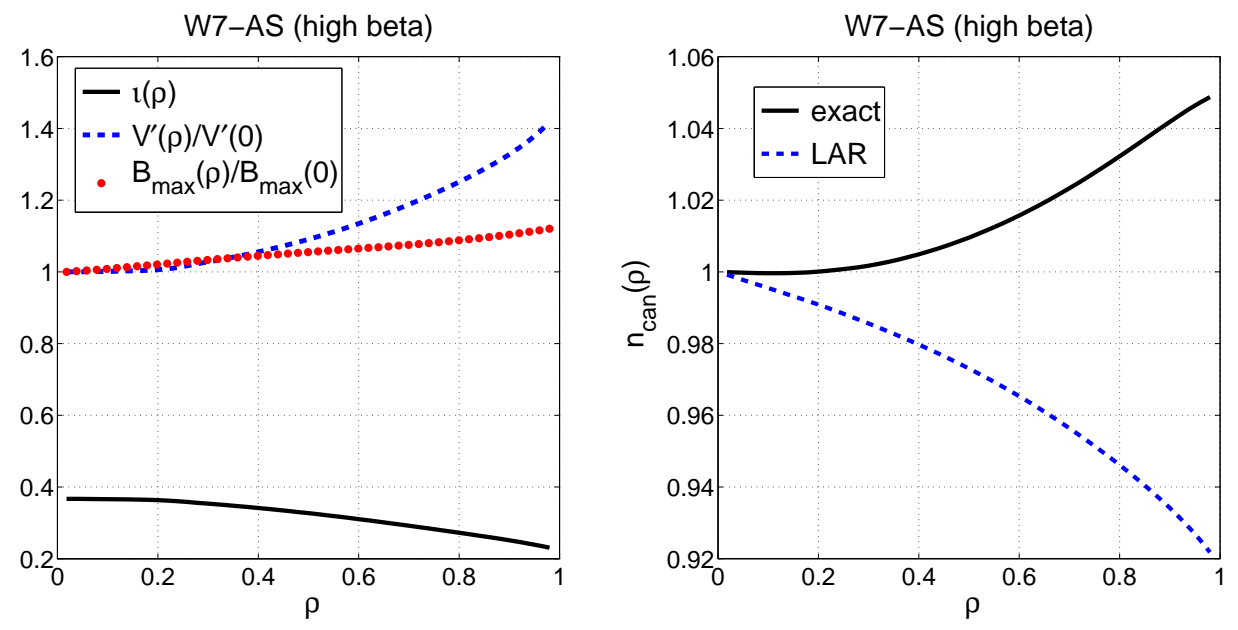

Figure 8: Mishchenko et al. 

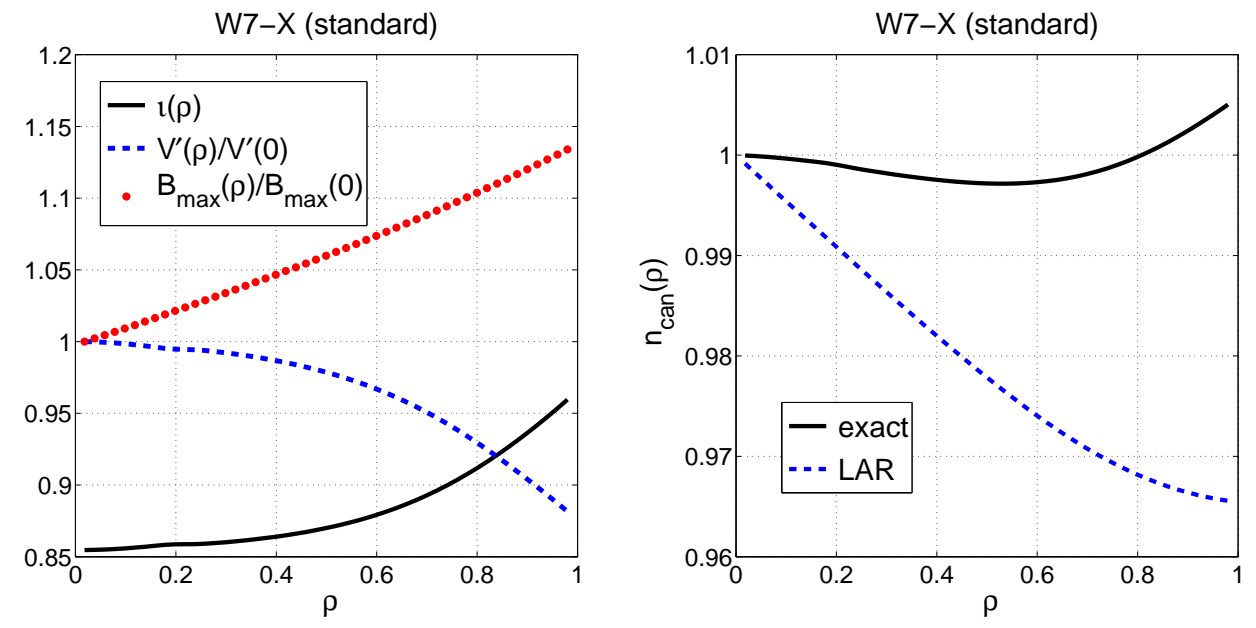

Figure 9: Mishchenko et al. 

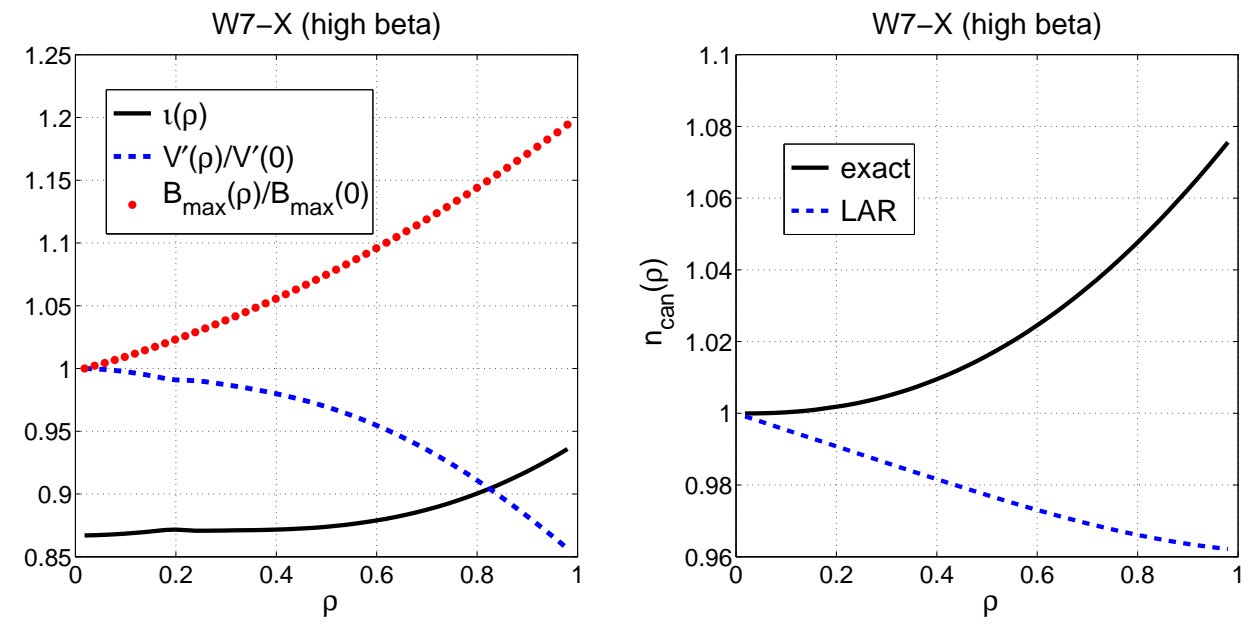

Figure 10: Mishchenko et al. 


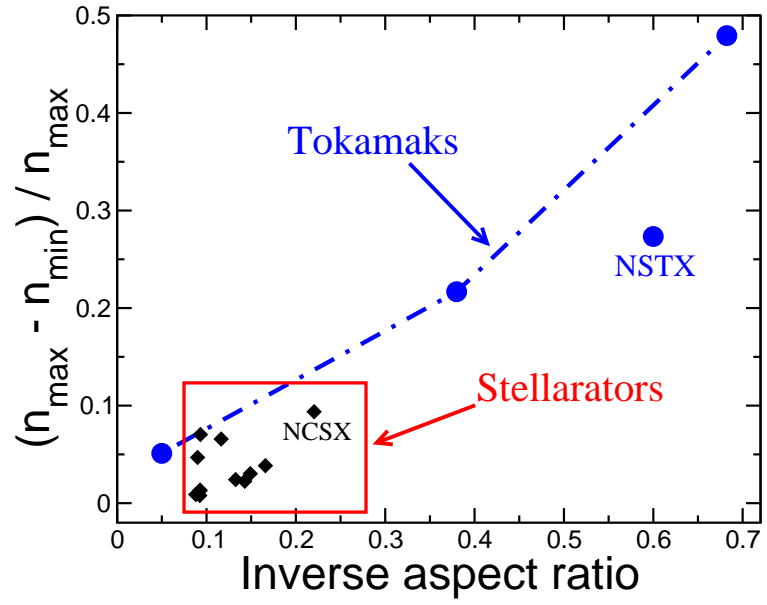

Figure 11: Mishchenko et al. 


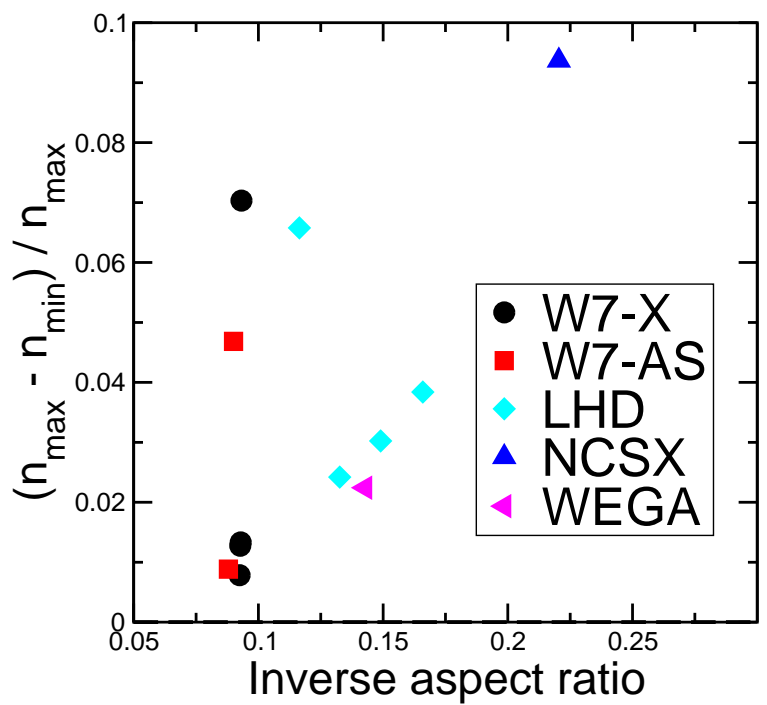

Figure 12: Mishchenko et al. 